\title{
Tissue transglutaminase in HCV infection
}

\author{
Cell Death and Differentiation (2003) 10, S79-S80. doi:10.1038/sj.cdd.4401112
}

Dear Editor,

Transglutaminases (TGase) are a family of $\mathrm{Ca}^{2+}$-dependent enzymes that catalyse the post-translational modification of proteins at the level of lysine and glutamine residues. Indeed, these enzymes promote the formation of stable protein polymers and/or the covalent incorporation of polyamines into proteins. ${ }^{1-3}$ At least seven distinct types of transglutaminase have now been identified in mammals: TGase 1 (or TG K), TGase 2 (or TG C, or tTG), TGase 3 (or TG E), TGase $X$, coagulation factor XIII, band 4.2, and prostate TGase. Several important pathologies are associated with deficiency in transglutaminase gene products indicating the relevant biological function of these enzymes. ${ }^{4}$

The focus of this letter will be on one member of this gene family, the 'tissue' transglutaminase (tTG; E.C. 2.3.2.13) or type-II transglutaminase or transglutaminase C. This enzyme, in mammals, has a molecular weight of about $80 \mathrm{kDa}$ and is constitutively expressed in endothelial, mesangial and smooth muscle cells in adult tissues. ${ }^{5,6}$ tTGcatalysed protein crosslinking has been suggested to be involved in different biological processes and to have also acquired additional functions in cell adhesion and as a Gprotein in signalling. ${ }^{7}$ tTG is involved in programmed cell death where its activation results in the formation of large insoluble cellular protein polymers, which, by preventing macromolecular leakage from the dying cell, contributes to limitation of the inflammatory response. 6,8

A large group of neurodegenerative diseases have been characterised by the abnormal accumulation of intracellular crosslinked protein deposits, which contribute to the pathogenesis of these diseases; the protein deposits may in part result from inappropriate activation of tTG. Example of these pathologies are Huntington's and Alzheimer's diseases. ${ }^{9}$ These diseases show many similarities with the liver damage occurring in various forms of hepatitis characterised by the formation of both intracellular and extracellular stable protein aggregates, such as the Mallory bodies in alcoholic hepatitis. ${ }^{10}$ We have previously demonstrated that in fibrotic hepatic diseases, such as in Budd-Chiari, the high apoptotic rate is associated with tTG overexpression by hepatocytes and with an abnormal release of the enzyme into the extracellular matrix (ECM). ${ }^{11}$

tTG-mediated crosslinks has been hypothesised to play an important role in fibrotic diseases such as pulmonary fibrosis, renal interstitial fibrosis, parasitic liver fibrosis and liver cirrhosis. ${ }^{12-14}$ The tTG enzymatic reaction contributes to these pathologies by increasing the resistance of the deposited extracellular matrix to breakdown.

Fibrogenesis is a dynamic process leading to the accumulation of matrix protein in the extracellular space.
The fibrosis is considered as a programmed response to injury and is dynamic and reversible; when the ECM deposition becomes chronic and the hepatic regeneration is not efficient, the final hepatic cirrhosis is unavoidable. ${ }^{15}$

tTG has been suggested to play a role in the ECM organization in both normal and pathological conditions. A number of ECM components, such as collagens, fibronectin, fibrinogen, laminin, nidogen and transforming growth factor- $\alpha 1$ (TGF- $\alpha 1$ ) act as tTG substrates. ${ }^{12,16-18}$ In addition, several inflammatory mediators, including TNF- $\alpha$, TGF- $\beta$, IL- $1 \beta$, IL- 6 , involved in the fibrotic process and in apoptosis induction are known to regulate tTG expression in various biological settings. 4,12

Fibrotic tissue accumulation is a severe complication in many chronic infections. One of the most important etiological agents of a significant number of liver diseases including chronic hepatitis, liver cirrhosis and hepatocellular carcinoma is the hepatitis $\mathrm{C}$ virus $(\mathrm{HCV})$, discovered in 1989. ${ }^{19}$

In order to get further information on the involvement of tTG in fibrogenesis, we have analysed tTG expression in liver biopsies of a large number of $\mathrm{HCV}$-infected patients followed at INMI, IRCCS Lazzaro Spallanzani. We studied a pool of 60 patients, aged between 18 and 60 , representative of the various stages of the liver pathology, and of the different HCV genotypes. It is interesting to note that $\mathrm{TTG}$ expression correlated with the progression of liver disease. The enzyme was preferentially localized in the hepatic parenchyma bordering the portal tracts during the initial stages of hepatic histological alterations, while tTG was preferentially detected in ECM when fibrogenesis became the prominent phenomenon. A statistically significant correlation was found between TTG expression and the different stages of periportal necrosis evaluated using METAVIR score system $(P=0.04)$ (Figure $1 \mathrm{~A})$. A strong statistically significant relationship was also found between the amount of tTG in the liver and the stage of fibrosis $(P=0.005)$ (Figure 1B).

Fibrogenesis is a process regulated by different cellular and molecular mechanisms and increasing evidence indicates an important role of tTG in generating and remodelling the ECM. The high amount of the enzyme detected during the initial stage of the disease may act as a liver repair response to limit the inflammation; in fact, fibrogenesis can be seen as a protective phenomenon against inflammatory cell invasion. In later stages, when the ECM deposition is massive, the fibrotic tissue becomes prominent and the liver infection resolves in cirrhosis; in this case, tTG cross-linking might contribute to the stabilisation of the fibrosis acting as a pathogenetic event. 
utilised as a marker to monitor the evolution of the fibrotic process. Finally, the demonstration of a possible role of tTG in the activation of latent TGF- $\beta,{ }^{20}$ a major factor stimulating matrix synthesis, suggests that the enzyme may play a central role in regulation of these processes.

\section{Acknowledgements}

The authors would like to thank Dr Enrico Girardi for his statistical advice. The work was partially supported by grants from: European Community 'Apoptosis Mechanisms', AIRC, 'AIDS' project to M Piacentini and Ricerca Corrente and Finalizzata from Ministero Sanit.

B

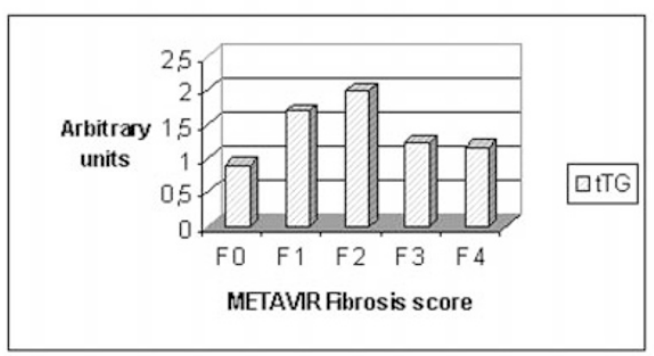

Figure 1 Comparison between tTG-positive hepatocytes and liver morphological index of METAVIR piecemeal necrosis score (PN) (A) and fibrosis score (F) (B) in HCV infected patients. Sixty liver specimens with different histological index of periportal infiltrate have been examined. Each section was semiquantitatively evaluated under code by three independent observers using a light microscope without knowledge of either clinical or histologica diagnosis. The degree of enzyme expression on each specimen, detected by immunohistochemistry, ${ }^{21}$ was expressed as arbitrary units comprised between 0 and 3 as follows: 0 negative staining; 1 weak positive staining; 2 positive staining; 3 strong positive staining. Continuous variables were expressed as mean-standard deviation (-SD) or as median (range), as appropriate. The Kruskal-Wallis test for non parametric data was used to compare medians; and the Pearson's was used to evaluate correlations. Statistical significance was set at $P>0.05$. The statistical analysis was performed by SPSS 10.0 for Windows (SPSS Inc. Chicago, IL, USA). Statistical analysis demonstrates a significant distribution of $\mathrm{TTG}$ in relation to the different stages of piecemeal necrosis $(P=0.04)(\mathbf{A})$, and in relation to the fibrosis stages $(P=0.005)(B)$

In conclusion, tTG plays an important role during hepatic fibrogenesis not only related to hepatitis $C$ infection but in many fibrogenetic pathologies. The strict correlation we observed between the TTG expression and the disease progression might imply that the enzyme expression can be
Roberta Nardacci ${ }^{1}$, Fabiola Ciccosanti ${ }^{1}$, Laura Falasca', Oreste Lo lacono ${ }^{1,3}$, Alessandra Amendola ${ }^{1}$, G Antonucci $^{1}$ and Mauro Piacentini, ${ }^{* 1,2}$

1 Istituto Nazionale Malattie Infettive, IRCCS L. Spallanzani, Rome, Italy

2 Department of Biology, University of Rome 'Tor Vergata', Rome, Italy

3 University of Palermo, Italy

* Corresponding author: Mauro Piacentini, Istituto Nazionale per le Malattie Infettive, IRCCS L. Spallanzani, Via Portuense 292, 00149 Rome, Italy. Tel: 3906-55170429; Fax: 3906-5594224;

E-mail: mauro.piacentini@uniroma2.it

1. Lorand L, Conrad SM (1984) Mol. Cell. Biochem. 58: 9-35

2. Folk JE, Finlayson S (1977) Adv. Protein Chem. 31:1-133

3. Greenberg CS et al. (1992) FASEB J. 5: 3071-3077

4. Aeschlimann D, Thomazy V (2000) Connective Tiss. Res. 41: 1-27

5. Thomazy V, Fesus L (1989) Cell Tissue Res. 255: 215-224

6. Piacentini M (1995) Curr. Top. Microbiol. 200: 163-176

7. Nakaoka $\mathrm{H}$ et al. (1994) Science 264: 1593-1596

8. Melino G, Piacentini M (1998) FEBS Lett. 430; 59-63

9. Lorand L (1996) Proc. Natl. Acad. Sci. USA 93: 14310-14313

10. Zatloukal K, et al. (1992) Lab. Invest. 66: 774-777

11. Piacentini M, et al. (1999) J. Pathol. 189: $92-98$

12. Mirza A, et al (1997) Am. J. Physiol. 272: G281-G288

13. Johnson TS, et al. (1997) J. Clin. Invest. 99: 2950-2960

14. Grenard P, et al. (2001) J. Hepatol. 35: 367-375

15. Friedman SL (2000) J. Biol. Chem. 275: 2247-2250

16. Kleman JP, et al. (1995) Biochemistry 34: 13768-13775

17. Aeschlimann D, Paulsson M (1991) J. Biol. Chem. 266: 15308-15317

18. Akimov SS, Belkin AM (2001) J. Cell Sci. 114: 2989-3000

19. Lauer GM, Walker BD (2001) N. Engl. J. Med. 345: 41-52

20. Kojima S, et al. (1993) J. Cell Biol. 121: 439-448

21. Castedo M, et al. (2001) J. Exp. Med. 194: 1097-1110 